\title{
Transient Anal Sphincter Relaxations Are a Normal Phenomenon in Healthy Subjects
}

TO THE EDITOR: Internal anal sphincter relaxation is an essential part of the sampling reflex ${ }^{1}$ and reflexes associated with defecation. ${ }^{2}$ Relaxation of the internal anal sphincter (IAS) during anorectal manometry can also occur "spontaneously," named spontaneous internal anal sphincter relaxation (SAR), when it is not associated with a stimulus. A recent study suggested that such an occurrence of a SAR indicates an "unstable IAS contraction" that "likely plays an important role in the development of fecal incontinence." ${ }^{3} \mathrm{Six}$ hundred and one patients with defecation disorders were assessed, only 23 of which exhibited SAR during anorectal manometry. The authors concluded that SAR was associated with neuropathy, ${ }^{3}$ but no evidence was presented that there is a causal relationship. The 23 patients were compared with 69 patients without SAR, randomly chosen from the same group. Interestingly, of the 23 patients that exhibited SAR, 11 were constipated and 12 were fecal incontinent, ${ }^{3}$ and most patients with fecal incontinence (FI) in their cohort did not display SAR. Clearly SAR is not a typical feature of FI. In a recent study on high-resolution colonic manometry spontaneous relaxations of the anal sphincter were observed in 10 of 19 healthy subjects. ${ }^{4}$ Furthermore, the anal sphincter often exhibited rhythmicity, therefore the relaxation phase can also be interpreted as a spontaneous relaxation. ${ }^{4}$ In addition, complete anal sphincter relaxation may occur in response to a spontaneous high amplitude propagating pressure wave that is not associated with defecation and happens without the subject being aware of it. ${ }^{4,5}$ Simultaneous pressure waves are also associated with anal sphincter relaxation, often associated with gas expulsion. ${ }^{6,7}$ In fact, any "spontaneous" relaxation may be due to a colonic motor event, ${ }^{4,6,8}$ hence a more appropriate term is "transient internal anal sphincter relaxation."

Taking all evidence into consideration, anal sphincter relaxations that are not associated with sampling or defecation are a com- mon feature in healthy subjects, not associated with abnormal bowel function. In a study by Farouk et al ${ }^{9}$ transient anal sphincter relaxations were common in patients with FI (7-12/hour) and healthy subject (3-7/hour), but fecal leakage was attributed to concurrent high rectal pressure and damage to the external anal sphincter. ${ }^{9}$ The conclusion of Lee et al ${ }^{3}$ that transient IAS relaxations can be used to assess morbidity and mortality risks in patients with anorectal disorders is therefore not warranted. To label a patient with transient IAS relaxations as having a neuropathy may lead to unnecessary concern for patients and unnecessary intervention.

Jan D Huizinga, Natalija Milkova, and Ji-Hong Chen* Farncombe Family Digestive Health Research Institute, McMaster University, Hamilton, ON, Canada

1. Bajwa A, Emmanuel A. The physiology of continence and evacuation. Best Pract Res Clin Gastroenterol 2009;23:477-485.

2. Bharucha AE. High amplitude propagated contractions. Neurogastroenterol Motil 2012;24:977-982.

3. Lee TH, Lee JS, Kim J, et al. Spontaneous internal anal sphincter relaxation during high-resolution anorectal manometry is associated with peripheral neuropathy and higher Charlson comorbidity scores in patients with defecatory disorders. J Neurogastroenterol Motil 2020;26:362-369.

4. Milkova N, Parsons SP, Ratcliffe E, Huizinga JD, Chen JH. On the nature of high-amplitude propagating pressure waves in the human colon. Am J Physiol Gastrointest Liver Physiol 2020;318:G646-G660.

5. Dinning PG, Bampton PA, Andre J, et al. Abnormal predefecatory colonic motor patterns define constipation in obstructed defecation. Gastroenterology 2004;127:49-56.

6. Chen J-H, Parsons SP, Shokrollahi M et al. Characterization of simultaneous pressure waves as biomarkers for colonic motility assessed by highresolution colonic manometry. Front Physiol 2018;9:1248.

7. Chen J-H, Yu Y, Yang Z, et al. Intraluminal pressure patterns in the human colon assessed by high-resolution manometry. Sci Rep 
2017;7:41436.

8. Pervez M, Ratcliffe E, Parsons SP, Chen JH, Huizinga JD. The cyclic motor patterns in the human colon. Neurogastroenterol Motil Published Online First: 02 Mar 2020. doi: 10.1111/nmo.13807.

9. Farouk R, Duthie GS, Pryde A, McGregor AB, Bartolo DC. Internal anal sphincter dysfunction in neurogenic faecal incontinence. Br J Surg
$1993 ; 80: 259-261$

Financial support: None.

Conflicts of interest: None.

Author contributions: Jan D Huizinga, Natalija Milkova, and JiHong Chen contributed to the manuscript writing. 